Pacific Journal of Mathematics

COUNTABLY COMPACT GROUPS AND FINEST TOTALLY 


\title{
COUNTABLY COMPACT GROUPS AND FINEST TOTALLY BOUNDED TOPOLOGIES
}

\author{
W. W. COMFORT AND VICTOR SAKS
}

The first main result formalizes the general principle that each totally bounded group $G$ is dense in some group $H$, not much larger than $G$, in which every subset of small cardinality has a complete accumulation point. For example: If $G$ is totally bounded and $|G|=\mathfrak{n} \geqq \aleph_{0}$, then $G$ is dense in a countably compact group $H$ such that $|H| \leqq \mathfrak{n}^{\aleph_{0}}$. A corollary: If $K$ is an infinite compact group with weight not exceeding $2^{\text {n}}$, then $K$ contains a dense, countably compact subgroup $H$ with $|H| \leqq \mathfrak{n}^{\boldsymbol{N}_{0}}$.

The following results are given in $\S 2$ : If $t$ is the finest totally bounded topological group topology on an infinite Abelian group $G$, then every subgroup of $G$ is $t$-closed and $(G, t)$ is not pseudocompact (both conclusions can fail for $G$ non-Abelian); a closed subgroup of a pseudocompact group need not be pseudocompact; if $\left\{\left(G_{i}, t_{i}\right\}: i \in I\right\}$ are nontrivial Abelian groups with their finest totally bounded topologies and $(G, \mathscr{T})$ is their product, then $\mathscr{T}=t$ if and only if $|I|<\aleph_{0}$.

1. Countably compact groups. Throughout this section the word group refers to a topological group which satisfies the Hausdorff separation axiom. Such spaces are known to be completely regular topological spaces. A group is said to be totally bounded if for each non-empty open subset $U$ of $G$ there is a finite subset $\left\{x_{k}: k<n\right\}$ of $G$ for which $G=\bigcup_{k<n} x_{k} \cdot U$. Each subgroup of a compact group is totally bounded, and Weil [26] has shown the converse: Each totally bounded group $G$ is (homeomorphic with) a dense subgroup of a compact group and this compactification is unique to within a topological isomorphism leaving $G$ fixed pointwise. We refer to this compactification of $G$ as the Weil completion of $G$, and we denote it by the symbol $\bar{G}$.

A completely regular Hausdorff space $X$ is countably compact if each of its infinite subsets has an accumulation point, and pseudocompact if each continuous, real-valued function on $X$ is bounded (equivalently: each locally finite family of open subsets of $X$ is finite). It is easy to see that each countably compact space is pseudocompact, and (as in [6], for example) that each pseudocompact group is totally bounded. Examples abound of pseudocompact groups which are not countably compact; see for example Kister [18] or H. Wilcox [28].

A number of theorems in the works of Itzkowitz [16], [17], and H. Wilcox [28] are devoted to showing that (in various settings and 
under various hypotheses) between a totally bounded group $G$ and its Weil completion $\bar{G}$ there is a pseudocompact group which is in a certain precise sense not much larger than $G$. The principal result of this section is a theorem of compactness type which has a number of corollaries improving these results. We show, specifically, that the groups of Itzkowitz and $\mathrm{H}$. Wilcox may be chosen countably compact.

Notation. If $m$ is a cardinal and $S$ is a set, then

$$
\mathscr{P}_{\mathrm{m}}(S)=\{A \subset S:|A|<\mathfrak{m}\} \text {. }
$$

If $m$ and $\mathfrak{n}$ are cardinals then

$$
\mathfrak{n}^{\mathfrak{m}}=\sum\left\{\mathfrak{n}^{\mathfrak{t}}: \mathfrak{t} \text { is a cardinal and } \mathfrak{t}<\mathfrak{m}\right\} .
$$

It is well-known and easy to prove that if $\mathfrak{m}$ and $\mathfrak{n}$ are infinite cardinals and $\mathfrak{m} \leqq \mathfrak{n}^{+}$, then

$$
\left|\mathscr{P}_{\mathfrak{m}}(\mathfrak{n})\right|=\mathfrak{n}^{\mathfrak{m}} \geqq \mathfrak{m} .
$$

(Here as usual, the symbol $\mathfrak{n}^{+}$denotes the smallest cardinal greater than $\mathfrak{n t}_{\text {.) }}$

Recall that if $X$ is a space and $Y \subset X$, then a point $p$ of $X$ is a complete accumulation point of $Y$ provided that

$$
|U \cap Y|=|Y|
$$

for each neighborhood $U$ of $p$ in $X$. It is obvious that if $X$ is compact, then each infinite subset of $X$ has a complete accumulation point in $X$.

The weight and density character of a space $X$ are denoted $w X$ and $d X$, respectively.

Definition. Let $\mathfrak{m}$ and $\mathfrak{n}$ be cardinal numbers with $\boldsymbol{\aleph}_{0} \leqq \mathfrak{m}<\mathfrak{n}$. The space $X$ is $[\mathrm{m}, \mathrm{n}]$-compact in the sense of complete accumulation points provided: If $Y \subset X$ and $\mathfrak{m} \leqq|Y|<\mathfrak{n}$, then $Y$ has a complete accumulation point in $X$.

The term we have just used is often defined as above except that it is required that $|Y|$ be a regular cardinal. Even this weaker property is strong enough to yield a compactness condition of covering type; in the interest of completeness we give a proof below. For positive results in the converse direction, see Alexandroff and Urysohn [2] and Aleksandrov [1], and for negative results in the converse direction see Mishchenko [21].

We note that in our terminology the spaces which are [ $\left[\boldsymbol{\aleph}_{0}, \mathbf{S}_{1}\right]$ compact in the sense of complete accumulation points are the countably compact spaces: each countably infinite subset has a (complete) accumulation point. 
Proposition. Let $\mathfrak{m}$ and $\mathfrak{n}$ be cardinal numbers with $\boldsymbol{\aleph}_{0} \leqq \mathfrak{m}<\mathfrak{n}$ and let $X$ have the property that if $Y \subset X$ and $\mathfrak{m} \leqq|Y|<\mathfrak{n}$ and $|Y|$ is regular then $Y$ has a complete accumulation point in $X$. Then for each open cover $\mathscr{U}$ of $X$ such that $\mathfrak{m} \leqq|\mathscr{C}|<\mathfrak{\pi}$ and $|\mathscr{C}|$ is regular, there is a cover $\mathscr{V} \subset \mathscr{Q}$ and $|\mathscr{V}|<|\mathscr{Q}|$.

Proof. Suppose that $\mathscr{C}=\left\{U_{\xi}: \xi<|\mathscr{C}|\right\}$ is a counterexample, and for $\xi<|\mathscr{C}|$ choose recursively $p_{\xi} \in X$ and $f(\xi)<|\mathscr{C}|$ as follows:

(i) $p_{0} \in X \backslash U_{0}$ and $p_{0} \in U_{f(0)}$;

(ii) if $p_{\eta}$ and $f(\eta)$ have been defined for all $\eta<\xi$, then

$$
p_{\xi} \in X \mid \bigcup_{\eta<\xi}\left(U_{\eta} \cup U_{f(\eta)}\right) \text { and } p_{\xi} \in U_{f(\xi)} \text {. }
$$

Then with $Y=\left\{p_{\xi}: \xi<|\mathscr{Q}|\right\}$ we have $|Y|=|\mathscr{C}|$ so there is a complete accumulation point $p$ of $Y$ in $X$. If $\eta$ is chosen so that $p \in U_{\eta}$, then there exists $\xi>\eta$ such that $p_{\xi} \in U_{\eta}$. This contradiction completes the proof.

THEOREM 1.1. Let $G$ be a totally bounded group such that $|G|=$ $\mathfrak{n} \geqq \boldsymbol{\aleph}_{0}$ and let $\mathfrak{m}$ be a regular cardinal for which $\mathfrak{m} \leqq \mathfrak{n}^{+}$. Then there is a group $H$, with $G \subset H \subset \bar{G}$, such that $H$ is $\left[\boldsymbol{\aleph}_{0}, \mathbf{m}\right]$-compact in the sense of complete accumulation points and $|H| \leqq \mathfrak{n}$.

Proof. For $A \in \mathscr{P}_{\mathrm{m}}(\widehat{G})$ with $|A| \geqq \aleph_{0}$ let $p_{A}$ be a complete accumulation of $A$ in $\bar{G}$, and for $S \subset \bar{G}$ let

$$
F(S)=S \cup\left\{p_{A}: A \in \mathscr{P}_{\mathrm{m}}(S),|A| \geqq \aleph_{0}\right\} ;
$$

and for $S \subset \bar{G}$ let $\langle S\rangle$ denote the subgroup of $\bar{G}$ generated by $S$.

Now let $H_{0}=G$ and $H_{1}=\left\langle F\left(H_{0}\right)\right\rangle$ and recursively, if $\xi<m$ and $H_{\eta}$ has been defined for $\eta<\xi$, let

$$
H_{\xi}=\left\langle F\left(\bigcup_{\eta<\xi} H_{\eta}\right)\right\rangle \text {. }
$$

We show by induction $\left|H_{\xi}\right| \leqq \mathfrak{n}^{m}$ for $\xi<\mathfrak{m}$. This is true for $\xi=$ 0 because

$$
\left|H_{0}\right|=\mathfrak{n} \leqq \mathfrak{n}^{m}
$$

and for $\xi=1$ because

$$
\left|F\left(H_{0}\right)\right| \leqq\left|\mathscr{P}_{\mathrm{m}}\left(H_{0}\right)\right|=\mathfrak{n}^{\mathfrak{m}} .
$$

If $\left|H_{\eta}\right| \leqq \mathfrak{n}^{\mathrm{m}}$ for $\eta<\xi$ then $\left|\bigcup_{\eta<\xi} H_{\eta}\right| \leqq|\xi| \cdot \mathfrak{n}^{\mathfrak{m}}=\mathfrak{n}^{\mathfrak{m}}$, so

$$
\left|F\left(\bigcup_{\mathfrak{r}<\hat{\xi}} H_{\eta}\right)\right| \leqq\left(\mathfrak{n}^{\mathfrak{m}}\right)^{\mathfrak{m}}=\mathfrak{n}^{\mathfrak{m}} ;
$$

this last equality holds because $\mathfrak{m}$ is regular (see Bachmann [3], pp. 
152-153). Thus $\left|H_{\xi}\right| \leqq \mathfrak{n}^{\mathfrak{m}}$.

Now let

$$
H=\bigcup_{\xi<\mathrm{m}} H_{\xi} \cdot
$$

Then $H$ is a group and $G \subset H \subset \bar{G}$ and

$$
|H| \leqq \sum_{\xi<\mathfrak{m}}\left|H_{\xi}\right| \leqq \mathfrak{m} \cdot \mathfrak{n}^{m}=\mathfrak{n}^{m} \text {. }
$$

And if $A \in \mathscr{P}_{\mathrm{m}}(H)$ with $|A| \geqq \boldsymbol{\aleph}_{0}$ then because $\mathfrak{m}$ is regular there is $\xi<\mathfrak{m}$ such that $A \subset H_{\xi}$, and we have

$$
p_{A} \in F\left(H_{\xi}\right) \subset H_{\xi+1} \subset H ;
$$

thus $H$ is $\left[\boldsymbol{\aleph}_{0}, \mathfrak{m}\right]$-compact in the sense of complete accumulation points.

Our first corollary, but with "pseudocompact" in place of "countably compact", was given in the general case by H. Wilcox [28] and earlier, for Abelian groups $G$, by Itzkowitz [16], [17].

COROLLARY 1.2. Let $G$ be a totally bounded group such that $|G|=$ $\mathfrak{n} \geqq \boldsymbol{\aleph}_{0}$. Then there is a countably compact group $H$ such that $G \subset$ $H \subset \bar{G}$ and $|H| \leqq \mathfrak{n}^{\aleph_{0}}$.

Proof. This follows from Theorem 1.1, upon taking $\mathfrak{m}=\boldsymbol{\aleph}_{1}$.

CoRollary 1.3. For each infinite cardinal $\mathfrak{n}$ there is a totally bounded group $H$ which is $\left[\mathbf{S}_{0}, \mathfrak{n}^{+}\right]$-compact in the sense of complete accumulation points but not compact, and for which $d H \leqq \mathfrak{n}$ and $|H| \leqq 2^{\mathrm{n}}$.

Proof. Let $K$ be the compact group $2^{2 n}$. According to a wellknown result of Hewitt [11] and Pondiczery [22] there is a dense subset $S$ of $K$ with $|S|=\mathfrak{n}$. Let $G$ be the subgroup of $K$ generated by $S$, so that $|G|=\mathfrak{n}$ and $K=\bar{G}$ by Weil's theorem. The result now follows from Theorem 1.1, upon taking $\mathfrak{m}=\mathfrak{n}^{+}$and noting that

$$
\mathfrak{n}^{\mathfrak{m}}=\mathfrak{n}^{\mathfrak{n}}=2^{\mathfrak{n}} .
$$

COROLlaRY 1.4. There is a separable, countably compact group which is not compact.

Proof. This is the case $\mathfrak{n}=\boldsymbol{S}_{0}$ of Corollary 1.3 .

COROLlaRY 1.5. Let $\mathfrak{n}$ be a cardinal and let $K$ be a compact group such that $\boldsymbol{\aleph}_{0} \leqq w K \leqq 2^{\mathrm{n}}$. If $\mathfrak{m}$ is a regular cardinal for which $\mathfrak{m} \leqq \mathfrak{n}^{+}$, then $K$ contains a dense subgroup $H$ which $i$ s $\left[\mathbf{\aleph}_{0}, \mathfrak{m}\right]$-compact 
in the sense of complete accumulation points such that $|H| \leqq \mathfrak{n}^{\mathfrak{m}}$.

Proof. According to Kuzminov [20] there is a continuous function $f$ from the compact group $2^{w K}$ onto $K$. (For an English-language proof that $K$ is the continuous image of $2^{n}$ for some cardinal $\mathfrak{n}$ when $K$ is compact and Abelian, see Hewitt and Ross [12], pp. 423-424. That $\mathfrak{n}$ may be chosen to be $w K$ follows from general topological considerations as in Engelking [9] p. 162.) Again by the theorem of Hewitt [11] and Pondiczery [22] there is a dense subset $D$ of $2^{w K}$ with $|D| \leqq \mathfrak{n}$. Then $f[D]$ is dense in $K$, so there is a dense subgroup $G$ of $K$ such that $|G|=\mathfrak{n}$. Then $K=\bar{G}$ by Weil's theorem, so the result follows from Theorem 1.1.

The following two corollaries, with "countably compact" in place of "pseudocompact", are given by H. Wilcox [28]. The first of these is given by Itzkowitz [16], [17] for the case in which $K$ is Abelian.

CoRollary 1.6. Let $\mathfrak{n}$ be a cardinal and let $K$ be a compact group such that $\boldsymbol{\aleph}_{0} \leqq w K \leqq 2^{\mathrm{n}}$. Then $K$ contains a dense, countably compact subgroup $H$ such that $|H| \leqq \mathfrak{n}^{\aleph_{0}}$.

Proof. This follows from Corollary 1.5, upon taking $\mathfrak{m}=\aleph_{1}$.

We note that Corollary 1.6 may be proved by appealing to Wilcox's theorem in place of the result of Kuzminov. If $K$ is given as in Corollary 1.6 and $H$ is a dense, pseudocompact subgroup of $K$ with $|H| \leqq \mathfrak{n}^{\aleph_{0}}$ (as afforded by $\mathrm{H}$. Wilcox [28]) then according to Corollary 1.2 above applied to the pair $(H, K)$ there is a countably compact group $H^{\prime}$ for which

$$
H \subset H^{\prime} \subset \bar{H}=K
$$

and $\left|H^{\prime}\right| \leqq\left(\mathfrak{n}^{\aleph_{0}}\right)^{\aleph_{0}}=\mathfrak{n}^{\aleph_{0}}$.

COROLLARY 1.7. Assume the generalized continuum hypothesis. If $\pi$ is a cardinal and $K$ is an infinite compact group such that $|K|=$ $2^{2 n}$, then there is a dense, countably compact subgroup $H$ of $K$ such that $|H| \leqq \mathfrak{n}^{\aleph_{0}}$.

Proof. It is known that $|K|=2^{w K}$. (A direct proof is given by H. Wilcox [27]. Earlier Hulanicki [14] [15], using essentially an argument of Čech and Pospísil [4], showed that $|K|=2^{\theta K}$ where $\theta K$ denotes the smallest cardinal which is the cardinality of a family $\mathscr{C}$ of open subsets of $G$ such that $|\cap \mathscr{U}|=1$. Since $\theta K=w K$-see Hewitt and Ross [13], pp. 99-100-we have again $|K|=2^{w K}$.) From 
the generalized continuum hypothesis it follows that $w K=2^{n}$, so Corollary 1.6 applies.

We have shown in this section that several of the pseudocompact groups considered in [28] may in fact be taken to be countably compact. We close with an example showing that not all of the conclusions of [28] may be strengthened in this manner.

We continue the notational convention used earlier: If $G$ is a group and $S \subset G$, then $\langle S\rangle$ denotes the subgroup of $G$ generated by $S$. For $x \in G$ we write $\langle x\rangle$ in place of $\langle\{x\}\rangle$.

Here and later the symbol $\boldsymbol{T}$ denotes the circle group

$$
\boldsymbol{T}=\{z: z \text { is a complex number and }|z|=1\},
$$

and $\boldsymbol{Q}$ is the "rational subgroup" of $T$-i.e.,

$$
\boldsymbol{Q}=\{z \in \boldsymbol{T}: \arg z \text { is rational }\} \text {. }
$$

Definition. Let $G$ be a group and $x \in G$. Then $x$ is a metric element of $G$ if $\operatorname{cl}_{G}\langle x\rangle$ is metrizable.

THeOREM 1.8. Let $M$ be the set of metric elements of the group $T^{\aleph_{1}}$. Then $M$ is not a countably compact group.

Proof. We have $\boldsymbol{Q} \subset \boldsymbol{T}$, and hence $\boldsymbol{Q}^{\boldsymbol{\aleph}_{1}} \subset \boldsymbol{T}^{\boldsymbol{\aleph}_{1}}$. It is easy to see that every element of $\boldsymbol{T}^{\boldsymbol{\aleph}_{1}}$ is the limit of a sequence of elements of $\boldsymbol{Q}^{\boldsymbol{\aleph}_{1}}$. (In detail: Let $p \in \boldsymbol{T}^{\boldsymbol{\aleph}_{1}}$ and for $\xi<\boldsymbol{\aleph}_{1}$ and each integer $n>0$ let $q_{\hat{s}}^{(n)}$ be chosen in $\boldsymbol{Q}$ so that

$$
\left|q_{\xi}^{(n)}-p_{\xi}\right|<1 / n \text {. }
$$

Then $q^{(n)} \in \boldsymbol{Q}^{\aleph_{1}}$, and $q^{(n)} \rightarrow p$.) Thus it suffices to show

(a) $Q^{\aleph_{1}} \subset M$; and

(b) $M \subsetneq T^{\aleph_{1}}$.

For (a) let $x \in \boldsymbol{Q}^{\aleph_{1}}$ and let $S \subset \boldsymbol{\aleph}_{1}$ have the property that $|S| \leqq$ $\boldsymbol{\aleph}_{0}$ and for each $\xi<\boldsymbol{\aleph}_{1}$ there is $\eta \in S$ such that $x_{\xi}=x_{\eta}$. We claim that the natural projection $\pi: \boldsymbol{T}^{\boldsymbol{\aleph}_{1}} \rightarrow \boldsymbol{T}^{S}$ is one-to-one on $\langle x\rangle$. If $x^{m} \neq$ $x^{n}$ there is $\xi<\boldsymbol{\aleph}_{1}$ for which $x_{\xi}^{m} \neq x_{\xi}^{n}$, and then choosing $\eta \in S$ such that $x_{\xi}=x_{\eta}$ have

$$
\left(\pi\left(x^{m}\right)\right)_{\eta}=\left(x^{m}\right)_{\eta}=\left(x^{m}\right)_{\xi} \neq\left(x^{n}\right)_{\xi}=\left(x^{n}\right)_{\eta}=\left(\pi\left(x^{n}\right)\right)_{\eta} ;
$$

thus $\pi\left(x^{m}\right) \neq \pi\left(x^{n}\right)$ and the claim is established.

We claim next that $\pi$ is one-to-one on the closure in $T^{\boldsymbol{\aleph}_{1}}$ of $\langle x\rangle$. Indeed if $p, q \in \mathrm{cl}\langle x\rangle$ with $p_{\xi} \neq q_{\xi}$ for some $\xi<\aleph_{1}$, then upon choosing $\eta \in S$ such that $x_{\xi}=x_{\eta}$ we note that the projections $\pi_{\xi}$ and $\pi_{\eta}$ from $T^{\aleph_{1}}$ onto $\boldsymbol{T}_{\xi}$ and $\boldsymbol{T}_{\eta}$ respectively agree on $x$, hence on $\langle x\rangle$, hence on 
cl $\langle x\rangle$, hence at $p$ and $q$. Thus

$$
(\pi(p))_{\eta}=\pi_{\eta}(p)=\pi_{\xi}(p)=p_{\xi} \neq q_{\xi}=\pi_{\xi}(q)=\pi_{\eta}(q)=(\pi(q))_{\eta},
$$

so $\pi(p) \neq \pi(q)$.

Thus $\pi$ is a one-to-one, continuous function from the compact group cl $\langle x\rangle$ into the metrizable group $\boldsymbol{T}^{S}$.

Thus the function $\pi$, when restricted to the compact group $\mathrm{cl}\langle x\rangle$, is a one-to-one continuous function into the metrizable group $\boldsymbol{T}^{S}$. This restricted function is then a homeomorphism, $\mathrm{cl}\langle x\rangle$ is metrizable, and $x \in M$. Assertion (a) is proved.

For (b) it suffices to cite from [12] pp. 407-408 the familiar fact that there exists $x \in T^{\aleph_{1}}$ such that $\mathrm{cl}\langle x\rangle=\boldsymbol{T}^{\boldsymbol{\aleph}_{1}}$. Since $\boldsymbol{T}^{\boldsymbol{\aleph}_{1}}$ is not metrizable, we have $x \in T^{\aleph_{1}} \backslash M$.

2. Finest totally bounded topologies. Throughout this section the word group refers simply to a non-empty set together with a multiplication and inversion satisfying the usual group axioms; no topology is assured. Topological groups are denoted by the symbols $(G, \mathscr{T}),(G, t)$ and the like. It is assumed that these satisfy the Hausdorff separation axiom.

It is known (see for example Dixmier [8], p. 296 ff.; Kurosh [19], p. 157; von Neumann [25]; T. Wilcox [29]; and Hewitt and Ross [12], pp. 348-351) that there are groups $G$ with the property that for no topology $\mathscr{T}$ on $G$ is $(G, \mathscr{T})$ a totally bounded topological group. But if $G$ is an Abelian group then, because there are sufficiently many homomorphisms from $G$ to the circle group $\boldsymbol{T}$ to separate points of $G$, the group $G$ may be embedded algebraically into a product of copies of $\boldsymbol{T}$ and therefore there is a totally bounded topology $\mathscr{T}$ on $G$ relative to which $(G, \mathscr{T})$ is a topological group. According to Comfort and Ross [5], the totally bounded group topologies on the Abelian groups $G$ are precisely the topologies induced on $G$ by point-separating group of homomorphisms into $\boldsymbol{T}$; the finest such topology is the one induced by the group of all such homomorphisms.

It is well-known [8] that if a (not necessarily Abelian) group $G$ admits a totally bounded group topology $\mathscr{T}$ then it admits a (necessarily unique) finest such topology. We denote this latter topology on $G$, when it exists, by the symbol $t$. It is not difficult to see that $(G, t)$ has the property that each homomorphism from $(G, t)$ to a totally bounded group is continuous. Indeed $t$ may be defined as follows: Let $\left\{\left(H_{i}, f_{i}\right): i \in I\right\}$ be a listing of all pairs $(H, f)$ with $H$ a totally bounded topological group and $f$ a homomorphism from $G$ onto a dense subset of $H$, and let

$$
e: G \longrightarrow P=\prod_{i \in I} H_{i}
$$


be defined by the rule

$$
(e x)_{i}=f_{i}(x) ;
$$

then $e$ is a one-to-one map because $(G, i d)$ is one of the pairs $\left(H_{i}, f_{i}\right)$, and $t$ is the topology induced on $G$ (more precisely: on $e[G]$ ) by $P$.

It is clear from the foregoing remarks that the finest totally bounded group topology $t$ on $G$ is characterized by the property that each homomorphism from $G$ to a totally bounded group is $t$-continuous.

In this section we prove that for each infinite Abelian group $G$ the topological group $(G, t)$ is not pseudocompact. This improves an observation made in 1.8 of [5]. We show also that a product of infinitely many nontrivial totally bounded Abelian topological groups does not have its finest totally bounded topology.

Lemma 2.1. Let $G$ be an Abelian group and $H$ a subgroup of $G$. Then $H$ is t-closed in $G$.

Proof. If $x \in G \backslash H$ then $H$ and $x H$ are different elements of $G / H$ so there is a homomorphism $\chi$ from $G / H$ into $T$ such that $\chi(x H) \neq 1$. If $\varphi$ denotes the natural mapping from $G$ onto $G / H$ then $\chi \circ \varphi$ is a homomorphism from $G$ to $\boldsymbol{T}$ and

$$
x \notin(\chi \circ \varphi)^{-1}(\{1\}) \supset H \text {. }
$$

The result now follows from the fact that $\chi \circ \phi$ is $t$-continuous, so that $(\chi \circ \varphi)^{-1}(\{1\})$ is a closed subset of $(G, t)$.

Theorem 2.2. Let $G$ be an infinite Abelian group. Then $(G, t)$ is not a pseudocompact topological group.

Proof. It is well-known and easy to prove from standard structure theorems (see for example [12], page 227) that there is a subgroup $H$ of $G$ such that $|G / H|=\boldsymbol{\aleph}_{0}$. If $(G, t)$ were pseudocompact then $G / H$ in the usual quotient topology would be pseudocompact (being the continuous image of $G$ ), a Hausdorff space (because $H$ is closed by Lemma 2.1), and countable. Since $G / H$ is a pseudocompact, Lindelöf space it is countably compact ([10], Exercise $3 \mathrm{D})$; indeed, it is compact ([10], Theorem 8.2 and Exercise $5 \mathrm{H})$. But this is impossible, since an infinite countably compact group has cardinality at least $2^{\aleph_{0}}([12]$, page 31).

REMARKS 2.3. (a) An early version of this paper showed only that $(G, t)$ as in Theorem 2.2 could not be countably compact, and left unsettled the question whether $(G, t)$ might be pseudocompact. 
We are grateful to Lew Robertson for formulating the argument given above, which shows in effect that a pseudocompact group never contains a closed, normal subgroup of countably infinite index.

(b) If the word Abelian is omitted from Lemma 2.1 or from Theorem 2.2, the resulting statements are false. It has been pointed out to us by Lew Robertson that according to a result of van der Waerden [24] the real, special orthogonal group $\mathrm{SO}(3, \boldsymbol{R})$, which is an infinite, compact, connected, Lie group, admits no discontinuous homomorphism into any compact group. (It follows from (22.13), (22.14), and (22.22.h) of [12] that the complex special linear group SL(2,C) admits no algebraic isomorphism, continuous or discontinuous, into any compact group. Such a group is said to be minimal almost periodic; see [25].) According to the discussion preceding 2.1, then, this compact, metric topology on the (non-Abelian) group $\mathrm{SO}(3, \boldsymbol{R})$ is the finest totally bounded topology $t$ on $\mathrm{SO}(3, \boldsymbol{R})$. Since $\mathrm{SO}(3, \boldsymbol{R})$ contains copies of $\boldsymbol{T}$-and hence also non-closed copies of $\boldsymbol{Q}$-not every subgroup of $\mathrm{SO}(3, \boldsymbol{R})$ is $t$-closed.

There is another property relating to finest totally bounded topologies which, though it fails for the non-Abelian group $\mathrm{SO}(3, \boldsymbol{R})$, holds for each Abelian group: According to Theorem 2.2 those closed copies of $\boldsymbol{T}$ inside $\mathrm{SO}(3, \boldsymbol{R})$ do not inherit their own finest totally bounded topology. But if $H$ is any (necessarily $t$-closed) subgroup of a topological group $(G, t)$ with $G$ Abelian then the topology induced on $H$ is its finest totally bounded topology. To prove this it suffices, according to Theorem 1.7 of [5], to show that each homomorphism $f: H \rightarrow \boldsymbol{T}$ is continuous in this induced topology. Because $\boldsymbol{T}$ is divisible such a homomorphism $f$ extends to a homomorphism $\bar{f}: G \rightarrow T$; since $\bar{f}$ is $t$-continuous on $G$ its restriction to $H$ is also continuous.

Our next result answers a question suggested by 2.1 and 2.2. The construction follows an argument given in Theorem 2.3 of [28], and is clearly susceptible to substantial generalization.

THeorem 2.4. There is an Abelian pseudocompact group with a closed subgroup which is not pseudocompact.

Proof. Let $K=\boldsymbol{T}^{\boldsymbol{\aleph}_{1}}$ and let

$$
H=\left\{x \in K:\left|\left\{\xi<\boldsymbol{\aleph}_{1}: x_{\xi} \neq 1\right\}\right| \leqq \boldsymbol{\aleph}_{0}\right\} .
$$

( $H$ is an example of what Corson [7] calls a $\Sigma$-space.) That $H$ is countably compact is seen as in [7] or [18]: If $A \subset H$ and $|A| \leqq \boldsymbol{\aleph}_{0}$ then for some countable subset $S$ of $\aleph_{1}$ we have

$$
A \subset\left(\prod_{\xi \in S} T_{\xi}\right) \times \prod_{\xi \in \aleph_{1} \backslash S}\{1\}_{\xi} \subset H,
$$


so that each countable subset of $H$ is contained in a compact subspace of $H$.

Let $z \in \boldsymbol{T}$ have the property that $z^{n} \neq 1$ for each integer $n$ and let $p$ be that element of $K$ for which $p_{\xi}=z$ for all $\xi<\aleph_{1}$; and let $J$ denote the subgroup of $K$ generated by $p$.

Now let $G$ be the subgroup of $K$ generated by $H$ and $J$. Clearly (*) $\quad G=\left\{x \in K:\left|\left\{\xi<\boldsymbol{\aleph}_{1}: x_{\xi} \neq \boldsymbol{z}^{n}\right\}\right| \leqq \boldsymbol{\aleph}_{0}\right.$ for some integer $\left.n\right\}$.

We complete the proof by showing

(a) the group $G$ is pseudocompact;

(b) $J$ is a closed subgroup of $G$; and

(c) $J$ is not pseudocompact.

For (a) we note that $H$ is dense in $G$ (because $H \subset G \subset K$ and $H$ is dense in $K$ ) and that $H$ is countably compact and hence pseudocompact. Thus $G$ is pseudocompact.

For (b) we note that since the subgroup of $\boldsymbol{T}$ generated by $z$ is dense in $\boldsymbol{T}$, we have

$$
\operatorname{cl}_{K} J=\left\{x \in K: x_{\xi}=x_{\eta} \text { for all } \xi, \eta<\aleph_{1}\right\} .
$$

From $(*)$ and $(* *)$ it follows that

$$
\mathrm{cl}_{K} J \cap G=J,
$$

so that $J$ is closed in $G$.

For (c) we note that $J$ is (homeomorphic with) the group $\left\{z^{n} \in T: n\right.$ is an integer $\}$. This countable, infinite group is obviously not pseudocompact.

The proof is complete.

THeOREm 2.5. Let $\left\{\left(G_{i}, t_{i}\right): i \in I\right\}$ be a family of groups $G_{i}$ each with its finest totally bounded topology $t_{i}$. If $|I|<\boldsymbol{\aleph}_{0}$ and $(G, \mathscr{T})$ $i$ is the product of the spaces $\left(G_{i}, t_{i}\right)$, then the totally bounded topology $\mathscr{T}$ is the finest totally bounded group topology for $G$.

Proof. It suffices to treat the case $I=\{1,2\}$. Let $f$ be a homomorphism from $G=G_{1} \times G_{2}$ to a totally bounded group $H$, and let $U$ be a neighborhood in $H$ of the identity $e$ of $H$. Let $V$ be a neighborhood of $e$ such that $V^{2} \subset U$, and let $f_{1}$ and $f_{2}$ be defined from $G_{1}$ and $G_{2}$ respectively to $H$ by the rules

$$
f_{1}\left(g_{1}\right)=f\left(g_{1}, e_{2}\right), f_{2}\left(g_{2}\right)=f\left(e_{1}, g_{2}\right) .
$$

Because $t_{1}$ and $t_{2}$ are the finest totally bounded topologies on $G_{1}$ and $G_{2}$ respectively, the homomorphisms $f_{1}$ and $f_{2}$ are continuous. Thus there are neighborhoods $W_{1}$ and $W_{2}$ of the identity elements $e_{1}$ and $e_{2}$ 
such that

$$
f_{1}\left[W_{1}\right] \subset V \text { and } f_{2}\left[W_{2}\right] \subset V .
$$

It is now clear that

$$
f\left[W_{1} \times W_{2}\right] \subset V^{2} \subset U .
$$

We conclude that $f$ is continuous on $(G, \mathscr{T})$. Thus $\mathscr{T}$ is the finest totally bounded topology for $G$.

Our final result is in contrast with Theorem 2.5.

THEOREM 2.6. Let $\left\{\left(G_{i}, \mathscr{T}_{i}\right): i \in I\right\}$ be a family of totally bounded Abelian groups with $\left|G_{i}\right| \geqq 2$ for $i \in I$. If $|I| \geqq \aleph_{0}$ and $(G, \mathscr{T})$ is the product of the spaces $\left(G_{i}, \mathscr{T}_{i}\right)$, then the totally bounded topology $\mathscr{T}$ is not the finest totally bounded group topology for $G$.

Proof. Let $e_{i}$ be the identity element of $G_{i}$, and let

$$
H=\left\{x \in G:\left|\left\{i \in I: x_{i} \neq e_{i}\right\}\right|<\boldsymbol{\aleph}_{0}\right\} .
$$

Then $H$ is a dense, proper subgroup of $(G, \mathscr{T})$. The result now follows from Lemma 2.1.

\section{REFERENCES}

1. P. S. Aleksandrov, Some results in the theory of topological spaces, obtained within the last twenty-five years, Uspehi Mat. Nauk, 15, 2 (1960), 25-95 (= Russian Math. Surveys 15 No. 2 (1960), 23-83).

2. P. Alexandroff and P. S. Urysohn, Mémoire sur les espaces topologiques compacts, Verh. Akad. Wetensch. Amsterdam, 14 (1929), 1-96.

3. Heinz Bachmann, Transfinite Zahlen, Springer-Verlag, Berlin, 1955.

4. Eduard Čech and Bedřich Pospisil, I. Sur les espaces compacts, II, Sur les caractères des points dans les espaces $\mathscr{L}$, Publ. Fac. Sci. Univ. Masaryk 1938, No. 258, 14 pp. Also in Topological papers of Eduard Čech, 495-505, Publ. House Czech. Acad. Sci. Prague, 1968.

5. W. W. Comfort and K. A. Ross, Topologies induced by groups of characters, Fundamenta Math., 55 (1964), 283-291.

6. — Pseudocompactness and uniform continuity in topological groups, Pacific J. Math., 16 (1966), 483-496.

7. H. H. Corson, Normality in subsets of product spaces, Amer. J. Math., 81 (1959), 785-796.

8. J. Dixmier, Les $C^{*}$-algèbres et leurs representations, Gauthier-Villars, Paris, 1969.

9. R. Engelking, Outline of General Topology, North-Holland Publishing Company, Amsterdam, 1968.

10. Leonard Gillman and Meyer Jerison, Rings of Continuous Functions, D. Van Nostrand Company, Inc., Princeton, N. J., 1960.

11. E. Hewitt, A remark on density character, Bull. Amer. Math. Soc., 52 (1946), 641-643.

12. Edwin Hewitt and Kenneth A. Ross, Abstract Harmonic Analysis I, Springer-Verlag, Berlin, 1963. 
13. Edwin Hewitt and Kenneth A. Ross, Abstract Harmonic Analysis II, SpringerVerlag, Berlin, 1970.

14. A. Hulanicki, On cardinal numbers related with locally compact groups, Bull. Acad. Polon. Sci. Sér. Sci. Math. Astr. Phys., 6 (1558), 67-70.

15. - On the power of compact spaces, Colloq. Math., 7 (1960), 199-200.

16. G. L. Itzkowitz, Extensions of Haar measure for compact connected Abelian groups, Bull. Amer. Math. Soc., 71 (1965), 152-156.

17. - Extensions of Haar measure for compact connected Abelian groups, Indag. Math., 27 (1965), 190-207.

18. J. M. Kister, Uniform continuity and compactness in topological groups, Proc. Amer. Math. Soc., 13 (1962), 37-40.

19. A. G. Kurosh, The Theory of Groups, Vol. 2, Chelsea Publishing, New York, 1956.

20. V. Kuzminov, On a hypothesis of P. S. Aleksandrov in the theory of topological groups, Doklady Akad. Nauk SSSR, 125 (1959), 727-729.

21. A. S. Mishchenko, On finally compact spaces, Doklady Akad. Nauk SSSR, 145 (1962), 1224-1227 (= Soviet Math. Doklady 3 (1962), 1199-1202).

22. E. S. Pondiczery, Power problems in abstract spaces, Duke Math. J., 11 (1944), 835-837.

23. Victor Saks, Countably compact groups, Doctoral dissertation, Wesleyan University, 1972.

24. B. L. van der Waerden, Stetigkeitssätze für halbeinfache Liesche Gruppen, Math. Zeitschrift, 36 (1933), 780-786.

25. J. von Neumann, Almost periodic functions in a group, I, Trans. Amer. Math. Soc., 36 (1934), 445-492.

26. André Weil, Sur les espaces à structure uniforme et sur la topologie générale, Publ. Math. Univ. Strasbourg, Hermann, Paris, 1937.

27. Howard J. Wilcox, Pseudocompact groups, Doctoral dissertation, University of Rochester, Rochester, New York, 1965.

28. — Pseudocompact groups, Pacific J. Math., 19 (1966), 365-379.

29. Theodore W. Wilcox, On the structure of maximally almost periodic groups, Bull. Amer. Math. Soc., 73 (1967), 732-734.

Received July 7, 1972. Parts of this paper appeared in [23], written by the secondlisted author under the supervision of the first-listed author. We are grateful to the referee for several suggestions improving our exposition. The first author gratefully acknowledges support received from the National Science Foundation under grant NSFGP-39263.

WESLEYAN UNIVERSITY

AND

UNIVERSITY OF COSTA RICA 


\section{PACIFIC JOURNAL OF MATHEMATICS}

\section{EDITORS}

RICHARD ARENS (Managing Editor) University of California

Los Angeles, California 90024

R. A. Beaumon'T

University of Washington Seattle, Washington 98105
J. Dugundj1*

Department of Mathematics University of Southern California Los Angeles, California 90007

D. Gilbarg and J. Milgram Stanford University Stanford, California 94305

\section{ASSOCIATE EDITORS}

E. F. BECKENBACH

B. H. NeUMANN

F. WOLF

K. YOSHIDA

\section{SUPPORTING INSTITUTIONS}

UNIVERSITY OF BRITISH COLUMBIA CALIFORNIA INSTITUTE OF TECHNOLOGY UNIVERSITY OF CALIFORNIA MONTANA STATE UNIVERSITY UNIVERSITY OF NEVADA NEW MEXICO STATE UNIVERSITY OREGON STATE UNIVERSITY UNIVERSITY OF OREGON OSAKA UNIVERSITY

\section{UNIVERSITY OF SOUTHERN CALIFORNIA STANFORD UNIVERSITY UNIVERSITY OF TOKYO UNIVERSITY OF UTAH WASHINGTON STATE UNIVERSITY UNIVERSITY OF WASHINGTON AMERICAN MATHEMATICAL SOCIETY NAVAL WEAPONS CENTER}

* C. R. DePrima California Institute of Technology, Pasadena, CA 91109, will replace J. Dugundji until August 1974. 


\section{Pacific Journal of Mathematics}

\section{Vol. 49, No. $1 \quad$ May, 1973}

A. Bigard, Free lattice-ordered modules ...........................

Richard Bolstein and Warren R. Wogen, Subnormal operators in strictly cyclic

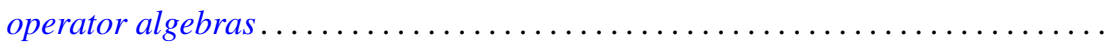

Herbert Busemann and Donald E. Glassco, II, Irreducible sums of simple

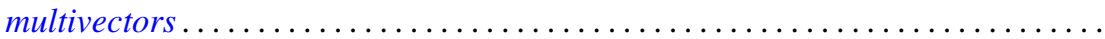

W. Wistar (William) Comfort and Victor Harold Saks, Countably compact groups

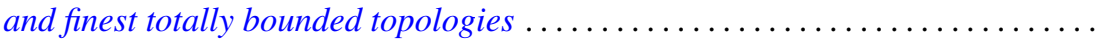

Mary Rodriguez Embry, Maximal invariant subspaces of strictly cyclic operator

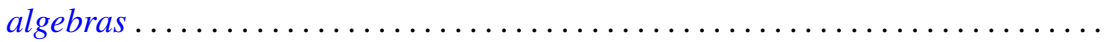

Ralph S. Freese and James Bryant Nation, Congruence lattices of semilattices......

Ervin Fried and George Grätzer, A nonassociative extension of the class of

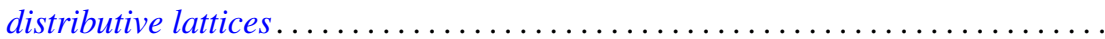

John R. Giles and Donald Otto Koehler, On numerical ranges of elements of locally

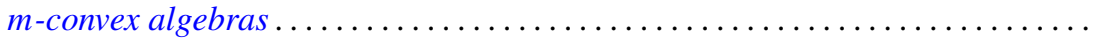

David A. Hill, On dominant and codominant dimension of $\mathrm{QF}-3$ rings ........ John Sollion Hsia and Robert Paul Johnson, Round and Pfister forms over $R(t) \ldots$ I. Martin (Irving) Isaacs, Equally partitioned groups . . . . . . . . . . . . . .

Athanassios G. Kartsatos and Edward Barry Saff, Hyperpolynomial approximation of solutions of nonlinear integro-differential equations.

Shin'ichi Kinoshita, On elementary ideals of $\theta$-curves in the 3-sphere and 2-links in the 4-sphere

Ronald Brian Kirk, Convergence of Baire measures

R. J. Knill, The Seifert and Van Kampen theorem via regular covering spaces ..

Amos A. Kovacs, Homomorphisms of matrix rings into matrix rings ..

Young K. Kwon, HD-minimal but no $H D$-minimal ..........

Makoto Maejima, On the renewal function when some of the mean renewal lifetimes are infinite

Juan José Martínez, Cohomological dimension of discrete modules over profinite groups.

W. K. Nicholson, Semiperfect rings with abelian group of units

Louis Jackson Ratliff, Jr., Three theorems on imbedded prime divisors of principal ideals.

Billy E. Rhoades and Albert Wilansky, Some commutants in $B(c)$ which are almost matrices

John Philip Riley Jr., Cross-sections of decompositions . . .

Keith Duncan Stroyan, A characterization of the Mackey uniformity $m\left(L^{\infty}, L^{1}\right)$ for

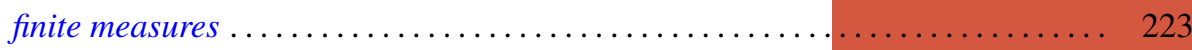

Edward G. Thurber, The Scholz-Brauer problem on addition chains . . . . . . . . . 229

Joze Vrabec, Submanifolds of acyclic 3-manifolds ............

Philip William Walker, Adjoint boundary value problems for compactified singular

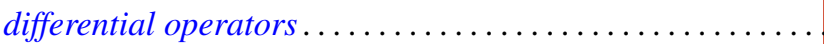

